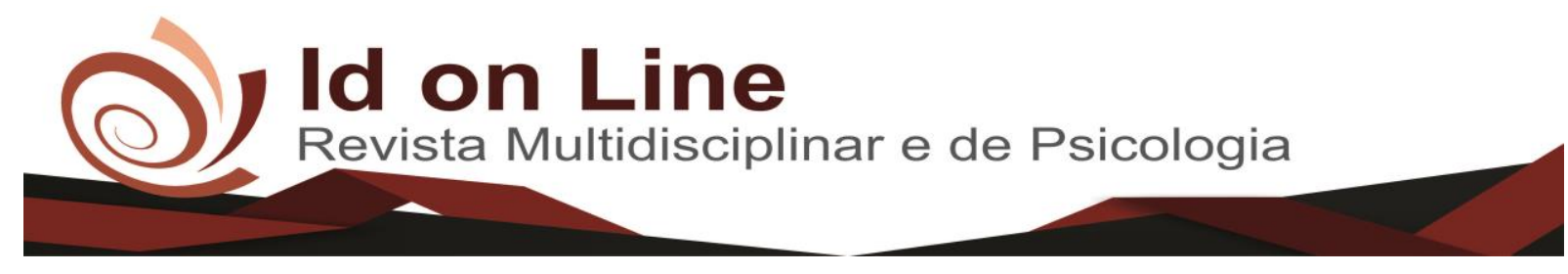

DOI: 10.14295/idonline.v15i55.3058

\title{
Ocorrência de Schistosoma mansoni (SAMBON, 1907) (Digenea: Schistosomatidae) em um Município Endêmico no Sul da Bahia
}

\author{
Midian Lima Araújo ${ }^{1}$, Jan Kleber de Oliveira Cardoso ${ }^{I}$, Danilla Rodrigues de Almeida ${ }^{1}$, \\ Lorena Aguiar Leite Dourado ${ }^{1}$, Viviane Amaral Toledo Coelho ${ }^{2}$, Carla Giselly de Souza ${ }^{3}$
}

\begin{abstract}
Resumo: A esquistossomose é uma endemia parasitária, típica das Américas, Ásia e África e atualmente ainda representa um problema de saúde pública. Estima-se que aproximadamente 25 milhões de pessoas ainda correm risco de contrair essa doença. Onde, os estados de maior incidência sãō Alagoas, Pernambuco, Sergipe, Minas Gerais, Bahia, Paraíba e Espírito Santo. Sua difusão ocorre principalmente pela precariedade e/ou ausência de saneamento básico. Deste modo, o objetivo desse estudo foi analisar a ocorrência da esquistossomose no distrito de Vila do Café, município de Encruzilhada - BA. A pesquisa foi realizada no distrito de Vila do Café, pertencente ao município de Encruzilhada-BA. Foram avaliadas 100 pessoas com idade entre 15 e 80 anos, sem distinção de gênero e os dados avaliados pelo método Kato-Katz- Dos 100 pacientes avaliados, $42 \%$ deram positivo para a patologia. Os dados desta pesquisa nos revelam que o distrito de Vila do Café, município de Encruzilhada - BA, é endêmico para ocorrência de esquistossomose. Diante disso, a atuação do poder público é indispensável no combate e prevenção ao Schistosoma mansoni, uma vez que a construção de uma infraestrutura sanitária adequada é um dever que cabe ao Estado.
\end{abstract}

Palavras-chaves: Esquistossomose; Saneamento básico; Saúde pública; Inquérito coproscópico.

\section{Occurrence of Schistosoma mansoni (SAMBON, 1907) (Digenea: Schistosomatidae) in an Endemic Municipality in Southern Bahia- Brazil}

\begin{abstract}
Schistosomiasis is a parasitic endemic, typical of the Americas, Asia and Africa and currently it still represents a public health problem. It is estimated that approximately 25 million people are still at risk of contracting this disease. Where, the states with the highest incidence are; Alagoas, Pernambuco, Sergipe, Minas Gerais, Bahia, Paraíba and Espírito Santo. Its diffusion occurs mainly due to precariousness and / or lack of basic sanitation. Thus, the objective of this study was to analyze the occurrence of schistosomiasis in the district of Vila do Café, municipality of Encruzilhada - BA. The research was carried out in the district of Vila do Café, belonging to the municipality of Encruzilhada-BA. 100 people aged between 15 and 80 years were evaluated, regardless of gender and the data evaluated using the Kato-Katz method. Of the 100 patients evaluated, $42 \%$ tested positive for the condition. The data of this research reveal that the district of Vila do Café, municipality of Encruzilhada - BA, is endemic for schistosomiasis. In view of this, the performance of the public authorities is indispensable in combating and preventing S. mansoni, since the construction of an adequate health infrastructure is a duty that belongs to the State.
\end{abstract}

Keywords: Schistosomiasis; Basic sanitation; Public health; Coproscopic survey.

\footnotetext{
${ }^{1}$ Faculdade de Almenara - ALFA, Curso de Farmácia. Almenara - MG - Brasil. alvesdasilvagr@gmail.com, jan_kleber@hotmail.com; danillarodriguesalmeida@hotmail.com; aguiarleitedourado@hotmail.com

${ }^{2}$ Faculdade de Almenara - ALFA, Professor. Almenara - MG - Brasil. E-mail: vivianeatc@yahoo.com.br;

${ }^{3}$ Universidade Federal da Grande Dourados-UFGD, Dourados/MS, Brasil. E-mail: carlaxlsouza@yahoo.com.br.
} 


\section{Introdução}

A esquistossomose se define como uma endemia parasitária, típica das Américas, Ásia e África. Os ovos do helminto S. mansoni (SAMBON, 1907) (Digenea: Schistosomatidae), que causa essa parasitose, foram encontrados há mais de dois mil anos em múmias chinesas. No século XXI, essa doença permanece como um problema grave de saúde pública. A Organização Mundial da Saúde (OMS) estabelece que a esquistossomose comprometa 200 milhões de pessoas em 74 países atualmente (KATZ; ALMEIDA, 2003).

No Brasil, estima-se que a esquistossomose, conhecida também como "barriga

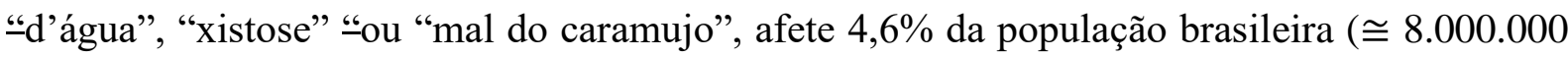
de pessoas), com maior prevalência nos habitantes de áreas rurais (SOUZA; VITORINO; COSTA, 2011). Aproximadamente 25 milhões de pessoas ainda correm risco de contrair essa doença.

O diagnóstico juntamente com as medidas profiláticas e o controle epidemiológico são extremamente importantes para o tratamento desses parasitos. A endemia atinge 19 estados brasileiros, apresentando maior incidência nos estados de Alagoas, Pernambuco, Sergipe, Minas Gerais, Bahia, Paraíba e Espírito Santo. Sua difusão e ocorrência estão ligadas à precariedade e/ou ausência de saneamento básico (FREITAS et al., 2013).

A região Nordeste e o estado de Minas Gerais foram as primeiras áreas endêmicas da esquistossomose no Brasil (KATZ; ALMEIDA, 2003). Na Bahia, a doença é notificada em 65\% (271/417) dos municípios, com média de 165,8 internações/ano, 40,2 óbitos/ano e prevalência de 5,4\% (CARDIM et al., 2011; FARIAS et al., 2011). Apesar de mais de 30 anos de ações de controle, a Bahia constitui uma elevada prevalência, inquéritos coproscópicos realizados pela Fundação Nacional de Saúde (FUNASA), entre 1986 e 1994 apontaram uma prevalência média de 9,6\% no estado (FARIAS et al., 2011).

A esquistossomose é uma doença causada claramente por condições socioeconômicas adversas ou omissas, diretamente ligadas à falta de infraestrutura- de armazenamento e distribuição adequada de água e saneamento básico, fatores que evitariam que as populações residentes em zonas endêmicas tivessem contato direto com águas contaminadas por caramujos - hospedeiro intermediário do parasito (FONSECA et al., 2013).

O distrito de Vila do Café, distante $35 \mathrm{~km}$ da sede Encruzilhada - BA e $8 \mathrm{~km}$ da cidade de Mata Verde - MG, é cercado por riachos que foram por muito tempo utilizados pelas mulheres para lavagem de roupas e utensílios domésticos, e pelos trabalhadores para lavagem 
de automóveis e por muitas crianças para banho no período de verão. Povoado por aproximadamente 12 mil habitantes, o distrito não dispõe de saneamento básico como água tratada e instalação de rede de esgoto, fator predeterminante para ocorrência dessa parasitose na região. Nos últimos anos, um número considerável de pessoas morreu com ascite, popularmente conhecida como "barriga d'água", em decorrência da patologia (IBGE, 2020).

Visto os dados alarmantes da prevalência dessa parasitose, principalmente no estado da Bahia, sejam pelas baixas condições socioeconômicas quanto pela total ausência de saneamento básico, o objetivo desse estudo foi analisar a ocorrência da esquistossomose no distrito de Vila do Café, município de Encruzilhada - BA-e estabelecer uma relação entre a precariedade dos serviços de saneamento básico e ações de saúde com a prevalência dessa parasitose na região e sua morbidade e comorbidade.

\section{Material e Métodos}

A pesquisa foi realizada no distrito de Vila do Café, pertencente ao município de Encruzilhada-BA, localizada na mesorregião centro-sul, a 607 km de Salvador. O município é cercado por riachos que são utilizados domesticamente pela população e também para irrigação de hortaliças ou consumo individual. Cerca de 23.766 mil habitantes (IBGE, 2020).

A Secretaria Municipal de Saúde do município em questão, realizou a coleta de dados para controle anual da parasitose e forneceu os dados para o presente estudo, por isso não foi necessário a submissão ao Comitê de Ética (dados secundários). Foram avaliadas 100 pessoas com idade entre 15 e 80 anos, sem distinção de gênero e os dados avaliados pelo método KatoKatz. Na Técnica de Kato-Katz as fezes são coletadas; a fresco, em coletor universal e processadas dentro de 24 horas. Foram realizadas três lâminas por paciente. Todo o material foi examinado ao microscópio e feita a contagem dos ovos, realizado em laboratório do município (KATZ; CHAVES; PELEGRINO, 1972).

Os resultados foram dispostos de acordo com a idade, sexo e infecção pelo parasito. Os indivíduos infectados foram notificados e encaminhados para a unidade de saúde onde foram avaliados pelo profissional da área que estabeleceu o tratamento específico. 


\section{Resultados}

No município foram analisadas 100 pessoas, 51 eram do sexo feminino e 49 do sexo masculino. Foi possível observar que, 34 deram positivo para Schistosoma mansoni.

Pode-se observar que o percentual de ovos do parasito esquistossemese no sexo masculino foi predominante, devido às condições de trabalhos rurais, que ocasiona maiores exposições e contato com o parasito, compreendendo $64,7 \%$ de contaminação na população, onde, dos 49 homens avaliados, 22 eram positivos e 23 negativos. No sexo feminino, observouse $35,3 \%$ de contaminação, das 51 mulheres avaliadas, 12 foram positivos e 35 negativos. Além disso, dos 100 indivíduos avaliados, $8 \%$, (4 homens e 4 mulheres) testaram positivos para outras parasitoses (Figura 2).

Conforme pode ser observado na Figura 1, a faixa etária mais acometida foi entre 20 e 40 anos. Ou seja, a população mais ativa, seja pela idade ou economicamente.

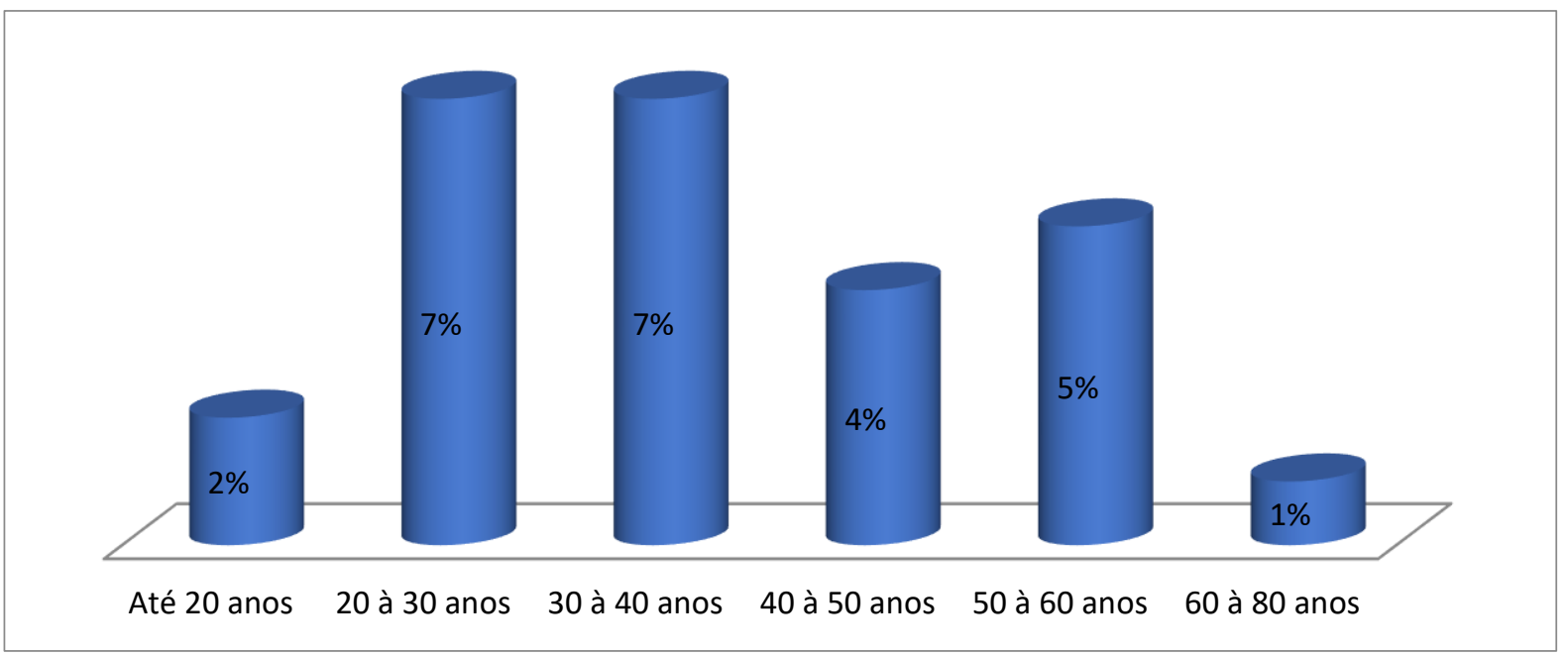

Figura 1 - Infecção por Schistosoma mansoni (SAMBON, 1907) de acordo com a faixa etária das 100 pessoas avaliadas, no distrito de Vila do Café, município de Encruzilhada, BA.

Ao desdobrar; o resultado para outras parasitoses, foi verificado que, além dos 34 casos de S. mansoni, a presença de parasitos como cinco casos de Ancylostoma duodenale, dois de Trichuris trichiura e dois de Ascaris lumbricoides (Figura 2). 


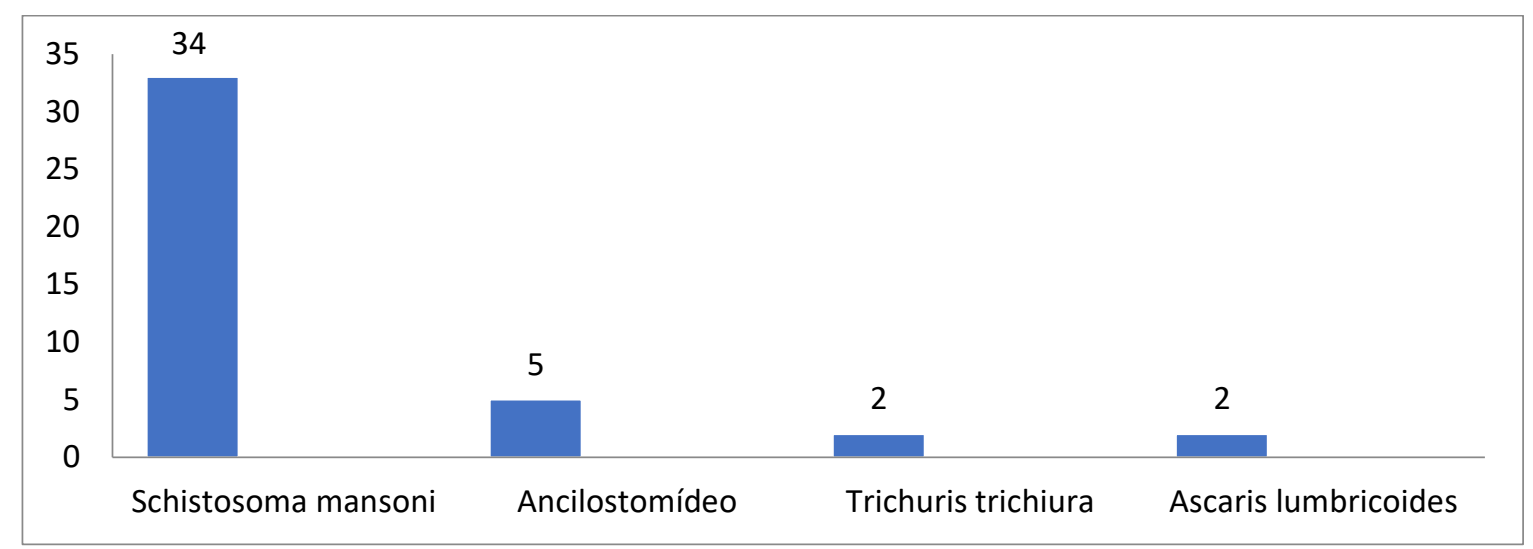

Figura 2 - Ocorrência de ovos de Schistosoma mansoni (SAMBON, 1907) e de outros parasitos em amostras de fezes de 100 habitantes avaliados no distrito de Vila do Café, município de Encruzilhada, BA-

Além disso, dos 100 indivíduos analisados, foi verificado um caso de poliparasitismo, ou seja, a presença de mais de um parasito (S. mansoni, A. lumbricoides e A. duodenale).

\section{Discussão}

A esquistossomose é considerada um grave problema de saúde pública em todo o mundo, sendo associada à pobreza, aos hábitos culturais e ao nível socioeconômico de uma população (CARDIM et al., 2011; PALMEIRA et al., 2010). O fato de estar diretamente ligada com a pobreza e a falta de higiene faz com que a doença não desperte tanto interesse público, e consequentemente da comunidade científica, tão pouco dos grandes laboratórios farmacêuticos, uma vez que o mercado não é um alvo economicamente viável (CUNHA, 2012).

É importante ressaltar que o crescente número de casos registrados no Brasil nos últimos anos, não se refere somente ao crescimento das infecções, mas pode mostrar números que antes não eram notificados pela falta de um sistema de informação e coleta desses dados. Segundo Cunha (2012), o início do trabalho com o Sistema de Informação de Agravos de Notificação (SINAN), os casos de esquistossomose tiveram um aumento considerável, pois este órgão passou a registrar as informações a respeito dessa parasitose.

O SINAN, órgão operacionalizado no nível administrativo das unidades de saúde, segue a orientação de descentralização do SUS. Suas notificações são digitadas nas Secretarias Municipais de Saúde e contabilizadas no sistema nacional. Sendo alimentado, principalmente 
pela notificação e investigação de casos de doenças e agravos que constam da lista nacional de doenças de notificação compulsória, como é o caso da esquistossomose. Sua utilização efetiva, segundo o SINAN (BRASIL, 2006) proporciona a realização do diagnóstico na população investigada, podendo fornecer subsídios para explicações causais dos agravos de notificação compulsória, além de vir a indicar riscos aos quais as pessoas estão sujeitas, contribuindo assim, para a identificação da realidade epidemiológica de determinada área geográfica.

O uso sistemático de forma descentralizada auxilia para a democratização da informação, onde todos os profissionais de saúde podem ter acesso à informação, sendo então, um instrumento relevante para auxiliar o planejamento da saúde, definir prioridades de intervenção, além de permitir que seja avaliado o impacto das intervenções (BRASIL, 2010).

Em relatos sobre a distribuição da esquistossomose segundo percentual de positividade em inquéritos coproscópicos - Brasil, 2012 mostra que a área ocupada por Rubim (Vale do Jequitinhonha) possui uma faixa de positividade entre 5 a 15\% (BRASIL, 2014).

Por conta da grande quantidade de casos ainda registrados no Brasil, o Ministério da Saúde sugere que as cidades possam tratar as comunidades pertencentes às áreas de maior risco, buscando reduzir a transmissão da infecção e suas possíveis complicações e intensificar a busca de melhorias nas condições de saneamento. O Programa de Controle da Esquistossomose, criado em 1980, veio para incentivar as ações de controle, a mobilização social, a educação em saúde e a inserção dos dados gerados no sistema de informação correspondente. Para isso, recomendação é a realização das atividades de vigilância e de controle da esquistossomose nos municípios mediante a incorporação das ações do Programa às desenvolvidas na Atenção Primária em Saúde, realizadas pela Estratégia de Saúde da Família dos municípios (BRASIL, 2014).

Apesar de todos os esforços para o registro dos casos e tratamento, ainda existem dificuldades na execução das ações direcionadas ao controle da esquistossomose nos serviços de saúde diante da precariedade de funcionamento dos serviços públicos de não só na cidade de Rubim, mas também em todo o Brasil (BRASIL, 2014).

Os problemas de saneamento básico e a falta apoio dos gestores e a pactuação da esquistossomose nas ações de vigilância em saúde podem atrapalhar o controle da doença. Por isso deve-se promover a sensibilização e responsabilização da comunidade a respeito do combate da infecção nessas áreas endêmicas e exercê-lo por meio do controle social (GIOVANELLA, 2006).

As atividades de controle e conscientização da esquistossomose são pouco abordadas pelas equipes da Saúde da Família e que são de grande importância para as ações de controle

359 Id on Line Rev. Mult. Psic. V.15, N. 55, p. 354-362, Maio/2021 - ISSN 1981-1179

Edição eletrônica em http://idonline.emnuvens.com.br/id 
da esquistossomose. O trabalho de Quites et al. (2016) com 97 profissionais mostrou que as ações da Estratégia de Saúde da Família (ESF) foram consideradas incipientes nos municípios do Vale do Jequitinhonha, MG. Destacam-se a ausência de estratégias específicas por parte da ESF para combater e controlar a infecção em áreas endêmicas, o recebimento insuficiente de material explicativo pelas equipes e a ausência de reuniões entre os profissionais da equipe da ESF para discutir assuntos relacionados à infecção em área endêmica.

Os moradores da cidade em questão têm o hábito de se banhar em rios e cachoeiras da região, sendo que esses locais são fontes de contaminação, podendo estar infectados com as formas larvais de S. mansoni. Por esses motivos, pode-se afirmar que a doença ainda afeta grande parte da população brasileira, especialmente as mais carentes.

Os dados acima abordados e a história mostram que ao invés de existir um processo linear e relativamente contínuo de transmissão epidemiológica das afecções humanas, no qual as chamadas doenças da pobreza são substituídas pelos males atuais, o que é observado é um quadro de interações complexas com mudanças, adaptações, e emergências peculiares dos fenômenos vivos que muitas vezes se mostram diferentes do esperado. A ligação entre as populações de homens, vetores e agentes etiológicos é realmente vasta e não aparenta estar no horizonte, para os anos futuros, a miragem de uma vida livre de infecções (FONSECA et al., 2013).

De forma geral, é necessário conscientizar os pacientes em tratamento dos benefícios do tratamento, efeitos colaterais e mostrar a necessidade de ingerir a quantidade de doses prescritas para o sucesso da terapia. A função do farmacêutico pode ser vista como uma ferramenta que contribui uma melhor escolha terapêutica para todos, auxiliando para a informação adequada sobre o tratamento da doença. É possível afirmar que mais de 200 milhões de pessoas estejam infectadas com espécies de Schistosoma (BROOKS et al., 2012).

Dessa forma, podemos constatar que a esquistossomose é uma patologia que afeta um grande número de pessoas pelo Brasil, principalmente em regiões onde o saneamento básico é inexistente ou precário. Diante disso, a atuação do poder público é indispensável no combate e prevenção ao $S$. mansoni, uma vez que a construção de uma infraestrutura sanitária adequada é um dever que cabe ao Estado. Além disso, as medidas precisam ser efetivas, uma vez que devem ser tratados ambos os hospedeiros e não apenas um como tem sido feito. É necessário reduzir o risco de exposição fazendo o controle de moluscos, isto é, combatendo a população de caramujos, principal ator na transmissão dessa infecção.

Porém, é importante ressaltar que a educação em saúde também se constitui em uma das principais medidas profiláticas efetivas no tocante à essa patologia, uma vez que a 
conscientização quanto aos hábitos de higiene, exames periódicos e o conhecimento em relação à endemia local pode mudar indicadores atuais.

\section{Conclusão}

Os dados desta pesquisa nos revelam que o distrito de Vila do Café, município de Encruzilhada - BA, é endêmico para ocorrência de esquistossomose. Diante disso, a atuação do poder público é indispensável no combate e prevenção ao Schistosoma mansoni, uma vez que a construção de uma infraestrutura sanitária adequada é um dever que cabe ao Estado.

Além disso, medidas sanitárias governamentais precisam ser efetivas, uma vez que devem ser tratados ambos os hospedeiros e não apenas um como tem sido feito. Investindo em saneamento básico, tratando os doentes e combatendo a população de caramujos, principal ator na transmissão dessa infecção no caso dessa parasitose.

\section{Referências}

ALMEIDA, L. M; KATZ, N. F. In: CIMERMAN B. Parasitologia humana e seus fundamentos gerais. $2^{a}$ ed. São Paulo: Atheneu; 2003.

BRASIL. Ministério da Saúde. Secretaria de Vigilância em Saúde. Guia de vigilância epidemiológica. $6^{a}$ ed. Brasília: Ministério da Saúde, 2009.

BRASIL. Ministério da Saúde. Secretaria de Vigilância em Saúde. Departamento de Vigilância Epidemiológica. Sistema de Informação de Agravos de Notificação-Sinan: normas e rotinas / Ministério da Saúde, Secretaria de Vigilância em Saúde, Departamento de Vigilância Epidemiológica. - Brasília: Editora do Ministério da Saúde, 2006.

BRASIL. Ministério da Saúde. Secretaria de Vigilância em Saúde. Departamento de Vigilância Epidemiológica. Vigilância da Esquistossomose Mansoni: diretrizes técnicas / Ministério da Saúde, Secretaria de Vigilância em Saúde, Departamento de Vigilância das Doenças Transmissíveis. - 4. ed. - Brasília: Ministério da Saúde, 2007.

BRASIL. Ministério da Saúde. Secretaria de Vigilância em Saúde. Departamento de Vigilância Epidemiológica. Vigilância da Esquistossomose Mansoni: diretrizes técnicas / Ministério da Saúde, Secretaria de Vigilância em Saúde, Departamento de Vigilância das Doenças Transmissíveis. - 4 ed. - Brasília: Ministério da Saúde, 2014.

BROOKS. G.F. et al., Microbiologia Medica de Jawetz, Melnick e Adelbert. 25. ed. AMGH, 813 p. Porto Alegre, 2012.

CARDIM, L. L. et al., Análises espaciais na identificação das áreas de risco para a esquistossomose mansônica no Município de Lauro de Freitas, Bahia, Brasil. Cad. Saúde Pública, Rio de Janeiro, vol. 27 no. 5: p. 899-908, maio, 2011. 
CHAVES, A. et al., Estudo comparativo dos métodos coprológicos de Lutz, Kato-Katz e Faust modificado. Rev. Saúde pública, S. Paulo, 13:348-52, 1979.

CUNHA, M. E. P. Fatores Culturais na Transmissão da Esquistossomose Mansônica. Arquivos Brasileiros de Cardiologia, São Paulo, v. 8, n. 1, 2012.

FARIAS, L. M. M. et al., Os limites e possibilidades do Sistema de Informação da Esquistossomose (SISPCE) para a vigilância e ações de controle. Cad. Saúde Pública, Rio de Janeiro, vol. 27 no. 10: p. 2055-2062, out, 2011.

FONSECA, C. S. M. Dislipidemias: comorbidade nos diferentes estágios da esquistossomose mansônica. Resumos Expandidos do I CONICBIO / II CONABIO / VI SIMCBIO (v.2) Universidade Católica de Pernambuco - Recife - PE - Brasil - 11 a 14 de novembro de 2013.

FREITAS, J. et al., Esquistossomose, uma doença no contexto da saúde pública brasileira. Nov@ Revista Cientifica. Vol. 2, no. 2. 2013. Disponível em: <http://177.159.202.218:83/index.php/NOVA/article/download/52/56> Acesso em: 18 mar. 2020.

GIOVANELLA, L. A atenção primária à saúde nos países da União Europeia: configurações e reformas organizacionais na década de 1990. Caderno Saúde Pública, v. 22. n. 6, p. 951-963, 2006.

IBGE - Instituto Brasileiro de Geografia e Estatística -. Área territorial oficial. Resolução da Presidência do IBGE de $\mathbf{n}^{\circ} \mathbf{5}$ (R. PR-5/02), 2020. Disponível em: <https://cidades.ibge.gov.br/brasil/ba/encruzilhada/panorama acesso em 07/04/2020> Acesso em: 18 mar. 2020.

KATZ, N.; ALMEIDA, K. Esquistossomose, xistosa, barriga d'agua. Cienc. Cult. vol. 55 no. 1 São Paulo Jan./Mar 2003.

KATZ, N.; CHAVES, A.; PELLEGRINO, J. A simple device for quantitative stool tick smear tecnique in schistosomiasis mansoni. Rev. Inst. Med. Trop. São Paulo, v.14, p. 397-400, 1972.

PALMEIRA, D. C. C. et al. Prevalência da infecção pelo Schistosoma mansoni em dois municípios do Estado de Alagoas. Revista da Sociedade Brasileira de Medicina Tropical, v. 43, n. 3, p. 313-317, 2010.

QUITES, H. F. et al. Avaliação das ações de controle da esquistossomose na Estratégia de Saúde da Família em municípios do Vale do Jequitinhonha em Minas Gerais. Revista Brasileira de Epidemiologia, v. 19, n. 2, p. 375-389, 2016.

SOUZA, F. P. C.; VITORINO, R. R.; COSTA A. P. Esquistossomose mansônica: aspectos gerais, imunologia, patogênese e história natural. Rev. Bras. Clin. Med, v. 9, n. 4. 2011.

\section{Como citar este artigo (Formato ABNT):}

ARAÚJO, Midian Lima ; CARDOSO, Jan Kleber de Oliveira; ALMEIDA, Danilla Rodrigues de; DOURADO, Lorena Aguiar Leite; COELHO, Viviane Amaral Toledo; SOUZA, Carla Giselly de. Ocorrência de Schistosoma mansoni (SAMBON, 1907) (DIGENEA: SCHISTOSOMATIDAE) em um Município Endêmico no Sul da Bahia. Id on Line Rev.Mult. Psic., Maio/2021, vol.15, n.55, p. 354362, ISSN:1981-1179.

Recebido: 08/04/2021

Aceito: 16/04/2021 